\title{
Zoanthids (Anthozoa, Hexacorallia) from the Great Barrier Reef and Torres Strait, Australia: systematics, evolution and a key to species
}

\author{
W. J. Burnett ${ }^{1,2, *}$, J. A. H. Benzie ${ }^{2}$, J. A. Beardmore ${ }^{1}$, J. S. Ryland ${ }^{1}$ \\ ${ }^{1}$ School of Biological Sciences, University of Wales Swansea, Singleton Park, Swansea SA2 8PP, UK \\ ${ }^{2}$ Australian Institute of Marine Science, PMB \# 3, Townsville M.C., Qld 4810, Australia
}

Accepted: 29 January 1996

\begin{abstract}
Zoanthid taxonomy is currently in a state of chaos, with many described species very few of which can be reliably identified. As part of a genetically based, objective reappraisal of the number of northern Australian species, a total of 355 zoanthid specimens were collected from 19 localities in the Great Barrier Reef and Torres Strait during 1992-1994. Specimens, initially assigned to one of ten morphological or ecological forms, were subjected to allozyme electrophoretic analysis. Analysis of genetic data revealed only seven discrete (i.e., non-interbreeding) groups in the family Zoanthidae. These groups, which are delimited by fixed gene differences, are considered species under a biological species concept. Some species show considerable morphological variation and have broad environmental tolerances. We provide the first key to Great Barrier Reef zoanthid species based on our results and observations. Species can be differentiated in the field on the basis of gross colony morphology, sand encrusting habit, polyp form and habitat. Genetic data are also used to generate a phylogenetic hypothesis of relationships among the Zoanthidae, which is compared to previous morphologically based systems. Division of the group on the basis of mesenterial arrangement appears justified, but nematocyst data appear less valuable in phylogenetic studies of the group than has been suggested previously.
\end{abstract}

\section{Introduction}

The Zoanthidea (Anthozoa, Hexacorallia) are a relatively little studied order of mainly colonial cnidarians, common throughout the tropics. Some species, particularly Palythoa spp. and Zoanthus spp., occupy large amounts of

Correspondence to: W. J. Burnett

* Present address: Scottish Association for Marine Science, PO Box 3, Oban, Argyll PA34 4AD, UK space on reef flats and crests (Karlson 1983; Muirhead 1989; Ryland and Muirhead 1993; Burnett 1995b; Burnett et al. 1995). The taxonomy of zoanthids is badly in need of revision. Identification has previously been certain only to generic level (Ryland and Muirhead 1993). Taxonomic reviews have historically been confounded by high levels of morphological variation within and between supposed species. Of the four families within Zoanthidea, the Zoanthidae are the most ubiquitous and important group on the Great Barrier Reef, and hence the most in need of revision. Sixty species of Zoanthidae are described from the central Indo-west Pacific (Walsh and Bowers 1971) but the true number is unknown. At least 25 are described or reported from the Great Barrier Reef and Torres Strait regions of northeast Australia (Hertwig 1882, 1888; Haddon and Shackleton 1891; Saville-Kent 1893; Wilsmore 1909; Carlgren 1937; Ryland and Muirhead 1993). From their descriptions many of these species appear to be identical, or are distinguished on the basis of characters more recently shown to have no taxonomic significance (Muirhead 1989).

In the present study, a genetic approach was used to evaluate the number of zoanthid species present in northeast Australia. Specimens were first placed into loose morphological or ecological groups, and the genetic integrity of these groups was then tested using allozyme electrophoresis. Groups which appeared to be genetically indistinguishable were merged, while those which appeared to contain genetic subgroups (individuals or groups of individuals with unusual allele combinations) were split. Discrete genetic groups of zoanthids which occur sympatrically were considered to be reproductively isolated from one another, and hence to be biological species. (We subscribe to Mayr's 1992 view that biological species concepts are meaningful in almost all systems including mixed sexual and asexual breeders and those groups which routinely hybridise, such as many plants and cnidarians.) Electrophoretic data have previously helped in the resolution of similar taxonomic problems in other Cnidaria, notably Actiniaria (Carter and Thorpe 1981; Bucklin and Hedgecock 1982; Shaw et al. 1987; Smith and Potts 1987; Solé-Cava et al. 1985; Solé-Cava 
and Thorpe 1987, 1992) and Scleractinia (Ayre et al. 1991; Weil 1992; Garthwaite et al. 1994; Stobart and Benzie 1994). This approach is not unlimited in its application, particularly since some closely related species may show very little or no differentiation at allozyme loci (Solé-Cava et al. 1985; Miller and Benzie in press). However it offers a valuable first assessment of zoanthid biodiversity in the Great Barrier Reef and Torres Strait and gives the first truly objective appraisal of morphological variation as an indicator of species identity in this group.

Allozyme data can be used to make phylogenetic inferences about the relatedness and evolution of taxa (Avise 1974; Felsenstein 1981; Buth 1984). Evolutionary studies of zoanthids have been hampered by the same problems as taxonomy, i.e. difficulties interpreting anatomical char- acters. There are no known fossils of zoanthids to cast any light on the history of the group (Wells and Hill 1956), but genetic data have the potential to offer valuable insights. This study used allozyme electrophoresis to determine species boundaries in Great Barrier Reef zoanthids and to examine evolutionary relationships within this group.

\section{Materials and methods}

355 zoanthid specimens were collected from 19 locations during 1992-1994 (Fig. 1). Specimens from each locality were initially assigned to one of ten morphological or ecological groups ("forms") in four genera, for a total of 45 'samples' (combinations of form and locality). For some forms it was possible to assign names based on

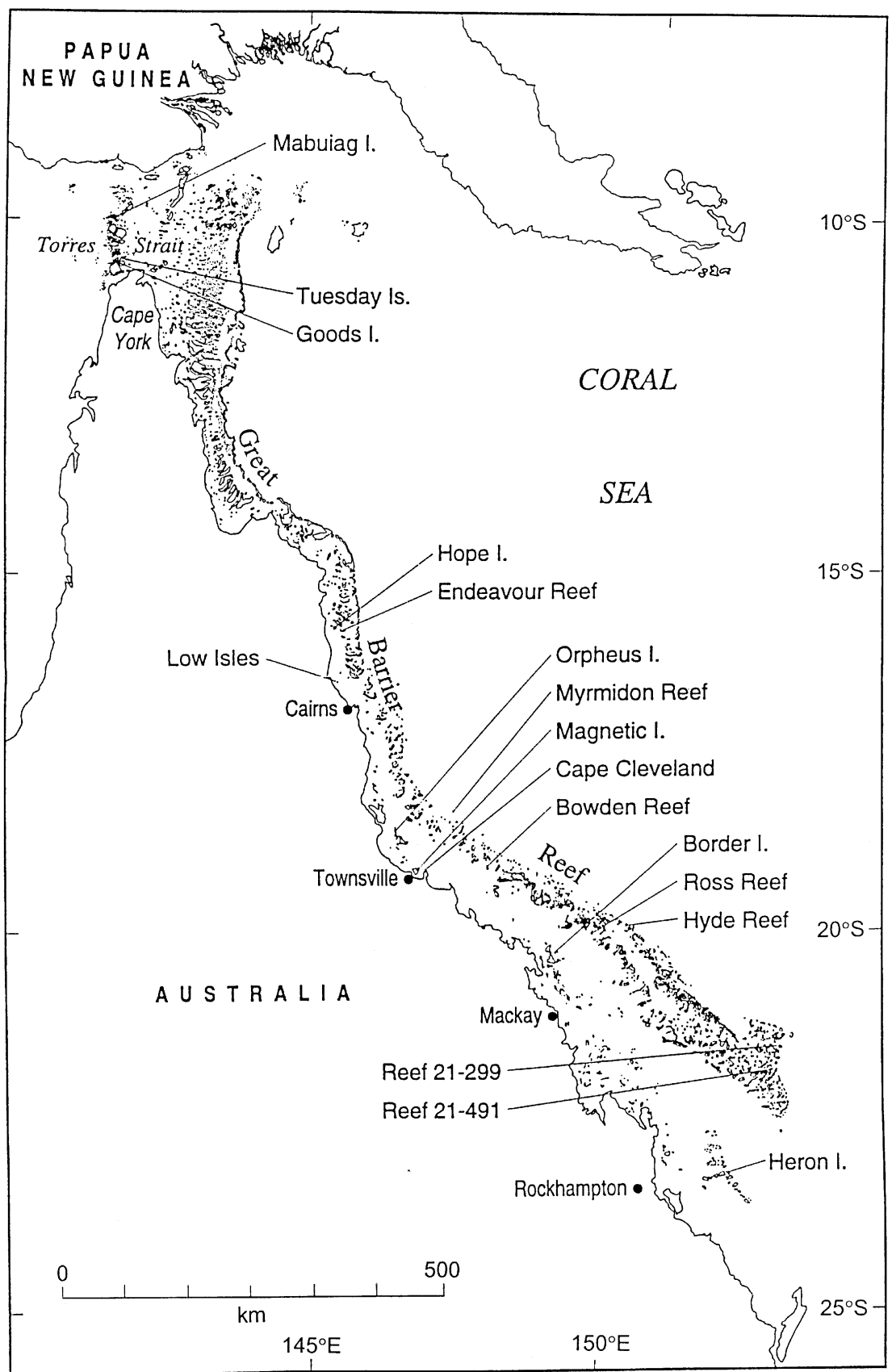

Fig. 1. Collecting sites in the Great Barrier Reef nd Torres Strait regions of north east Australia 
Table 1. Diagnostic features for 10 morphological groupings of Zoanthidae and one species (Parazoanthus dichroicus) of Parazoanthidae

\begin{tabular}{lll}
\hline Morphological group & Habitat & Generic features \\
\hline Palythoa caesia & $\begin{array}{l}\text { Subtidal on coral reefs. } \\
\text { Intertidal on reef crests }\end{array}$ & $\begin{array}{l}\text { Sediment encrusted polyps buried } \\
\text { in thick coenenchyme }\end{array}$
\end{tabular}

Form or specific features

Palythoa form $2 \quad$ Subtidal on coral reefs

Palythoa form $3 \quad$ Subtidal on coral reefs

Protopalythoa mutuki Intertidal, reef flats and rocky shores

Sediment encrusted polyps joined only at the base

Protopalythoa form 2 Subtidal on coral reefs

Sphenopus marsupialis Sandy or muddy substrata, seagrass beds

\section{Zoanthus coppingeri Intertidal, reef flats and rocky shores}

\section{Zoanthus form 2 Intertidal, reef flats and rocky shores}

Zoanthus form $3 \quad$ Subtidal on coral reefs

Zoanthus vietnamensis Intertidal on reef crests

Parazoanthus dichroicus Subtidal on coral reefs

Polyps small, encrusted with fine sediment. Ofter have yellow or orange tentacles

Large, solitary polyps free living in sand. Small polyps may be attached to stones or sea grass fronds

Polyps small, not sediment encrusted
Polyps large $(\sim 8 \mathrm{~mm}$ diameter $)$, colonies forming small ovoid blobs approx. $10 \mathrm{~cm}$ diameter or less. Generally buff coloured, ranging from almost white to dark brown

Polyps small ( $\sim 3 \mathrm{~mm}$ diameter) and tightly packed, colonies very large, up to at least $6 \mathrm{~m}$ diameter. Generally light brown

Polyps intermediate size, colonies forming irregular patches up to $50 \mathrm{~cm}$ diameter. Buff to dark brown

Intertidal. Polyps large, oral disk usually dark green with white mouth. Very heavily encrusted with large sand grains

Subtidal. Polyps relatively small unless growing out from under shade. Oral disk brown or green, frequently patterned

Polyps earthy grey

Forming extensive clonal patches, especially on unstable substrata (coral rubble) and the coral Montipora digitata. Colour bright and highly variable, generally red, orange, yellow or green

Disk with light coloured pattern on dark green or brown backround. Associated with Protopalythoa mutuki

Subtidal. Colonies usually small but occasionally extensive. Disk bright green., tentacles brown

Colonies thinly encrusting, polyps completely buried in coenenchyme. Forming extensive rubbery mats. Powder blue or blue/green

Growing on hydroids. Polyps small, beige, with distinct capitular ridges. Tentacles yellow or orange previous work (Burnett et al. 1994; Burnett 1995b; Burnett et al 1995), from species descriptions or following visits to type localities. Forms included three Palythoa morphs (P. caesia, P. form 2, P. form 3), two Protopalythoa morphs (Pro. mutuki, Pro. form 2), Sphenopus marsupialis and four Zoanthus morphs (Z. coppingeri, $Z$. form 2, Z. form $3, Z$. vietnamensis). A summary table of habitat and diagnostic features is given in Table 1. With the exception of Sphenopus marsupialis, specimens were photographed undisturbed in situ and carefully removed using a knife or hammer and chisel. A portion of each specimen was snap frozen in liquid nitrogen for genetic analysis, and the remainder fixed in $10 \%$ sea-water formalin for $48 \mathrm{~h}$, stored in $70 \%$ ethanol and kept for morphological examination. Specimens of Parazoanthus dichroicus Haddon and Shackleton, 1891, in the family Parazoanthidae, were collected from Bowden Reef for use as a genetic outgroup in phylogenetic analyses.

Fourteen allozyme loci coding for eleven different enzymes were screened using starch and cellulose acetate gels. The enzymes, buffer systems and running conditions used are shown (Table 2). Zymograms were visualised using stain recipes modified from Harris and Hopkinson (1976). Alleles were labelled according to their mobility relative to the most common allele in Palythoa caesia, which was assigned a mobility of 100 . Where multiple loci were detected for a single enzyme, loci were labelled numerically starting with that migrating fastest. Full details of specimen preparation, stain recipes and interpretation of allozyme banding patterns are given in Burnett (1995a)

Each specimen was initially checked to see whether it had any unusual alleles, or combinations of alleles, compared to the majority of specimens of that particular form in all samples. Individuals which carried rare alleles at any particular locus were not regarded as unusual if they also had a common allele, or had the same rare allele as another specimen which was heterozygous for a common allele. Unusual individuals were considered possible members of sibling species. This approach may have difficulty in identifying unusual individuals if the number of sibling species sampled approaches sample size, however this did not appear to be a problem with our data set. Of the 355 specimens analysed, two specimens of Protopalythoa form 2 from Ross Reef (Fig. 1) were considered genetically unusual. These two specimens shared GPI*, MDH-I* and $M E^{*}$ alleles with each other, but shared no alleles at these loci with any other Pro. form 2 specimens. These two specimens were redesignated Pro. form 3 and were considered a separate sample in further analyses.

The BIOSYS-1 computer package (Swofford and Selander 1981) was used to calculate gene frequencies from multi-locus genotypes, to perform exact tests for conformance to Hardy Weinberg predictions of genotype frequencies using Levene's (1949) correction for small sample size, and to calculate Nei's (1978) unbiased genetic distance (D) among samples. Exact tests were used as they are not 
Table 2. Enzyme/buffer systems employed for electrophoretic analysis of 14 putative enzyme loci

\begin{tabular}{lllll}
\hline Enzyme & $\begin{array}{l}\text { E.C. } \\
\text { number }\end{array}$ & No. loci & Buffer system & $\begin{array}{l}\text { Electrophoretic } \\
\text { medium }\end{array}$ \\
\hline Enolase (ENO) & 4.2 .1 .11 & 1 & Phos 7.0 & Cellulose acetate \\
Esterase D (EST-D) & 3.1 .1 .1 & 1 & TEC 7.9 & Starch \\
Glucose phosphate isomerase (GPI) & 5.3 .1 .9 & 1 & TEC 7.9 & Starch \\
Hexokinase (HK) & 2.7 .1 .1 & 1 & TEC 7.9 & Starch \\
Isocitrate dehydrogenase (IDH) & 1.1 .1 .42 & 1 & TEC 7.9 & Starch \\
LGG-Peptidase (LGG) & $3.4 .11 / 13$ & 2 & Poulik $^{\mathbf{c}}$ & Starch \\
LP-Peptidase (LP) & $3.4 .11 / 13$ & 1 & Poulik & Starch \\
LT-Peptidase (LT) & $3.4 .11 / 13$ & 2 & Poulik & Starch \\
Malate dehydrogenase (MDH) & 1.1 .1 .37 & 2 & TEC 7.9 & Starch \\
Malic enzyme (ME) & 1.1 .1 .40 & 1 & Phos 7.0 & Cellulose acetate \\
Phosphoglucomutase (PGM) & 5.4 .2 .2$. & 1 & TEC 7.9 & Starch \\
\hline
\end{tabular}

${ }^{a}$ Phos 7.0, continuous phosphate buffer, $\mathrm{pH} 7.0$ (Richardson et al. 1986)

b TEC 7.9, continous Tris-EDTA-Citrate buffer, pH 7.87 (Goodall and Stoddart 1989)

${ }^{c}$ Poulik. discontinous Boric acid-NaOH/Tris-Citrate, pH 8.2/8.7 (Poulik 1957) adversely affected by low expected values often encountered in small samples with rare alleles present (Lessios 1992). Significance values were corrected for multiple simultaneous tests (Miller 1980; Lessios 1992).

UPGMA cluster analysis of the 45 samples was performed using the PHYLIP computer package (Version 3.5c, Felsenstein 1993). Samples were assigned to one of seven discrete genetic groups, on the basis of genetic distance, the cluster analysis and fixed gene differences which indicated reproductive isolation of groups from one another. Each discrete genetic group was considered to represent a biological species.

Gene frequencies within species were pooled from all locations prior to phylogenetic analysis. This provided a conservative estimate of gene frequencies encompassing intraspecific variation within NE Australia. Alleles with frequencies less than 0.1 were combined with the most common allele at each locus for each genetic group. PHYLIP was then used to estimate a phylogeny using a maximum likelihood method (CONTML) which treats loci as characters with allele frequencies as unordered polymorphic states (Felsenstein 1981). The tree was constructed using the global optimisation option and ten random rearrangements of the species input order, using Parazoanthus dichroicus as an outgroup. The robustness of the phylogeny produced was estimated by bootstrapping 100 times using global optimisation. The number of random rearrangements for each bootstrap was kept to three to limit run time.

\section{Results}

Samples from all three forms of Palythoa appear to be genetically homogeneous with no fixed gene differences or clear differences in gene frequencies among them (Table 3a). In contrast, three genetic groups are apparent in Protopalythoa forms (Table 3b). Pro. mutuki is separated from Pro. form 2 by one fixed gene difference (at GPI*), and from Pro. form 3 by two fixed differences (at $M e^{*}$ and $H k^{*}$ ). Pro. forms 2 and 3 are separated by 3 fixed differences (at $G I^{*}, M D H-1^{*}$ and $M E^{*}$ ). There are major allele frequency differences at most loci. Gene frequencies of Zoanthus forms (Table 3c) show no major differences within or among samples of $Z$. coppingeri, $Z$. form 2 or $Z$. form 3. However $Z$. vietnamensis shows fixed differences from all other Zoanthus forms at seven loci $\left(E N O^{*}, L G G\right.$ $2^{*}, L P^{*}, L T-1^{*}, L T-2^{*}, M D H-1^{*}$ and $\left.M D H-2^{*}\right)$. Data for Sphenopus marsupialis and Parazoanthus dichroicus are unremarkable and are also shown in Table 3c.
There were no significant deviations from Hardy-Weinberg predictions of genotype frequencies in any sample using exact tests (data not shown). UPGMA cluster analysis of values of Nei's unbiased D among samples demonstrates seven genetic groups of Zoanthidae from the GBR, separated by fixed gene differences, and demonstrates their relatively distant relationship with Parazoanthus dichroicus (Fig. 2).

Samples of all three forms of Palythoa are mixed together on the UPGMA tree and genetic distances among samples are low (Fig. 2). There were no significant deviations from Hardy-Weinberg equilibrium if gene frequencies from the three different forms were pooled within localities, indicating no obvious departures from random mating among forms. The possibility remains that there may be undetected genetic differences among the three forms, and further study of the basis of morphological variation would clearly be worthwhile. For the purposes of this study, however, all three forms are considered to represent intraspecific variation in a single species, identified as $P$. caesia (Dana, 1846).

There are two highly unusual individuals among the Ross Reef Protopalythoa form 2. These two individuals show fixed gene differences from all other combined Pro. form 2 samples at three loci $\left(G P I^{*}, M D H-2^{*}, M E^{*}\right)$ and major gene frequency differences at several others. When treated as a separate population in the UPGMA analysis, they cluster closer to Pro. mutuki than to Pro. form 2, but fixed gene differences at $H K^{*}$ and $M E^{*}$ preclude their inclusion with Pro. mutuki. Values of D are lower within forms of Protopalythoa than between forms. We consider that our samples represent 3 species of Protopalythoa: Pro. mutuki Haddon and Shackleton, 1891, Pro. sp. 2 ( = form 2) and Pro. sp. 3 (= form 3 ).

No unusual populations or individuals were detected among the Sphenopus samples from three locations, and there is no evidence for the presence of more than one species. Specimens are all identified as Sphenopus marsupialis (Steenstrup, 1856).

Zoanthus coppingeri, $Z$. form 2 and $Z$. form 3 samples are mixed together in the UPGMA cluster analysis. Values of $\mathrm{D}$ are no higher between than within these forms and there are no fixed differences among them. When data 
Table 3a. Gene frequencies at 10 allozyme loci for 16 populations in three morphological groupings

\begin{tabular}{|c|c|c|c|c|c|c|c|c|c|c|c|c|c|c|c|c|}
\hline \multirow[b]{2}{*}{$\begin{array}{l}\text { Locus/ } \\
\text { Allele }\end{array}$} & \multicolumn{9}{|c|}{ Palythoa caesia } & \multicolumn{5}{|c|}{$P$. form 2} & \multicolumn{2}{|c|}{$P$. form 3} \\
\hline & Border & Hyde & $21-491$ & Low Is. & $\begin{array}{l}\text { Myrmi- } \\
\text { don }\end{array}$ & Orpheus & Ross & Tuesday & Hyde & Low & $\begin{array}{l}\text { Myrm- } \\
\text { idon }\end{array}$ & Orpheus & $21-491$ & Ross & Border & $\begin{array}{l}\text { Myrm- } \\
\text { idon }\end{array}$ \\
\hline \multicolumn{17}{|l|}{$E S T-D^{*}$} \\
\hline$(\mathrm{N})$ & (7) & (6) & (5) & (6) & (11) & (6) & (7) & (6) & (5) & $(5)$ & (14) & (7) & (3) & (6) & (5) & (15) \\
\hline 150 & - & - & - & - & 0.045 & - & - & - & - & - & - & - & - & - & - & - \\
\hline 142 & - & - & - & - & - & - & - & - & - & - & - & - & - & - & 0.100 & - \\
\hline 125 & 0.214 & 0.083 & 0.400 & 0.250 & 0.045 & 0.167 & 0.214 & 0.333 & 0.200 & 0.400 & 0.429 & 0.214 & 0.333 & 0.417 & 0.200 & 0.200 \\
\hline 100 & 0.786 & 0.917 & 0.500 & 0.750 & 0.909 & 0.667 & 0.786 & 0.333 & 0.800 & 0.600 & 0.571 & 0.786 & 0.500 & 0.583 & 0.700 & 0.800 \\
\hline 72 & - & - & 0.100 & - & - & 0.167 & - & 0.333 & - & - & - & - & 0.167 & - & - & - \\
\hline \multicolumn{17}{|l|}{$G P I^{*}$} \\
\hline 124 & - & - & - & - & - & - & - & - & - & - & 0.036 & 0.143 & - & 0.083 & - & - \\
\hline 119 & - & - & - & - & - & - & - & - & - & - & 0.071 & - & - & - & - & - \\
\hline 111 & 0.214 & 0.083 & - & 0.083 & 0.136 & 0.167 & - & 0.222 & 0.100 & 0.100 & 0.321 & 0.071 & - & 0.083 & 0.083 & 0.133 \\
\hline 100 & 0.786 & 0.917 & 1.000 & 0.917 & 0.864 & 0.833 & 1.000 & 0.611 & 0.900 & 0.800 & 0.571 & 0.714 & 1.000 & 0.750 & 0.917 & 0.800 \\
\hline 86 & - & - & - & - & - & - & - & 0.167 & - & 0.100 & - & 0.071 & - & 0.083 & - & 0.067 \\
\hline \multicolumn{16}{|l|}{$H K^{*}$} & $(15)$ \\
\hline 132 & - & - & 0.100 & - & - & - & - & - & 0.400 & - & - & - & - & - & - & 0.033 \\
\hline 126 & 0.286 & 0.333 & 0.300 & 0.417 & 0.227 & 0.417 & 0.071 & 0.167 & - & - & 0.143 & 0.214 & 0.333 & 0.333 & 0.250 & 0.200 \\
\hline 122 & - & - & - & - & - & - & - & - & - & - & - & - & - & - & - & 0.067 \\
\hline 115 & 0.357 & 0.250 & 0.300 & 0.167 & 0.364 & 0.167 & 0.071 & 0.333 & 0.300 & 0.700 & 0.357 & 0.071 & 0.167 & 0.167 & 0.167 & 0.267 \\
\hline 100 & 0.071 & 0.417 & 0.100 & 0.083 & 0.364 & 0.417 & 0.500 & 0.333 & 0.100 & 0.300 & 0.393 & 0.429 & 0.500 & 0.083 & 0.250 & 0.300 \\
\hline 87 & 0.214 & - & 0.200 & 0.250 & - & - & 0.214 & 0.167 & 0.200 & - & 0.107 & 0.214 & - & 0.333 & 0.167 & 0.100 \\
\hline 78 & 0.071 & - & - & 0.083 & - & - & 0.143 & - & - & - & - & 0.071 & - & 0.083 & 0.167 & 0.033 \\
\hline 66 & - & - & - & - & 0.045 & - & - & - & - & - & - & - & - & - & - & - \\
\hline \multicolumn{17}{|l|}{$I D H^{*}$} \\
\hline$(\mathrm{N})$ & (7) & (6) & (5) & (6) & (11) & (6) & (7) & (9) & (5) & (5) & (14) & (7) & (3) & (6) & (6) & (15) \\
\hline 110 & 0.143 & 0.167 & - & 0.083 & - & - & - & - & - & - & - & - & - & - & 0.250 & - \\
\hline 100 & 0.857 & 0.667 & 0.800 & 0.917 & 0.682 & 0.917 & 0.714 & - & 0.900 & 1.000 & 0.821 & 1.000 & 0.833 & 0.583 & 0.750 & 0.800 \\
\hline 90 & - & 0.167 & 0.200 & - & 0.318 & 0.083 & 0.286 & 0.833 & 0.100 & - & 0.107 & - & 0.167 & 0.417 & - & 0.200 \\
\hline 80 & - & - & - & - & - & - & - & 0.167 & - & - & 0.071 & - & - & - & - & - \\
\hline$L G G-1^{*}$ & & & & & & & & & & & & & & & & \\
\hline$(\mathrm{N})$ & (7) & (6) & (5) & (6) & (11) & (6) & (7) & (9) & (5) & (5) & (14) & (7) & (3) & (6) & (6) & (14) \\
\hline 122 & - & - & - & - & - & - & - & - & - & - & - & - & - & 0.083 & - & - \\
\hline 120 & - & - & - & - & 0.091 & - & - & 0.111 & 0.100 & - & 0.036 & 0.214 & 0.167 & - & - & 0.071 \\
\hline 112 & 0.429 & 0.500 & 0.400 & 0.250 & 0.318 & 0.417 & 0.214 & 0.222 & 0.300 & 0.500 & 0.321 & 0.286 & 0.333 & 0.500 & 0.500 & 0.286 \\
\hline 100 & 0.571 & 0.500 & 0.600 & 0.667 & 0.591 & 0.583 & 0.500 & 0.667 & 0.600 & 0.500 & 0.607 & 0.500 & 0.500 & 0.417 & 0.500 & 0.643 \\
\hline 96 & - & - & - & 0.083 & - & - & 0.286 & - & - & - & 0.036 & - & - & - & - & - \\
\hline$L P^{*}$ & & & & & & & & & & & & & & & & \\
\hline$(\mathrm{N})$ & (7) & (6) & (5) & (6) & (11) & (6) & (7) & (9) & $(5)$ & (5) & (14) & (7) & (3) & (6) & (6) & (14) \\
\hline 112 & 0.143 & 0.750 & 0.400 & 0.250 & 0.273 & 0.583 & 0.357 & 1.000 & 0.500 & 0.100 & 0.286 & 0.500 & 0.167 & 0.417 & 0.250 & 0.286 \\
\hline 100 & 0.857 & 0.250 & 0.600 & 0.750 & 0.727 & 0.417 & 0.643 & - & 0.500 & 0.900 & 0.714 & 0.500 & 0.833 & 0.583 & 0.750 & 0.714 \\
\hline$M D H-1$ & & & & & & & & & & & & & & & & \\
\hline$(\mathrm{N})$ & (7) & (6) & (5) & (6) & (11) & (6) & (7) & (9) & (5) & (5) & (14) & (7) & (3) & (6) & (6) & (15) \\
\hline 116 & 0.071 & 0.083 & - & 0.250 & 0.182 & - & - & 0.111 & - & 0.100 & 0.071 & 0.071 & 0.167 & - & - & - \\
\hline 100 & 0.929 & 0.917 & 1.000 & 0.750 & 0.818 & 0.917 & 1.000 & 0.889 & 1.000 & 0.900 & 0.893 & 0.929 & 0.833 & 1.000 & 1.000 & 1.000 \\
\hline 89 & - & - & - & - & - & 0.083 & - & - & - & - & 0.036 & - & - & - & - & - \\
\hline$M D H-2$ & & & & & & & & & & & & & & & & \\
\hline$(\mathrm{N})$ & (7) & (6) & (5) & $(6)$ & (11) & (6) & (7) & (9) & (5) & $(5)$ & (14) & (7) & (3) & (6) & (6) & (15) \\
\hline 105 & - & - & - & 0.083 & - & - & - & - & - & 0.200 & - & - & - & - & 0.083 & - \\
\hline 100 & 1.000 & 1.000 & 1.000 & 0.917 & 1.000 & 1.000 & 1.000 & 1.000 & 1.000 & 0.800 & 0.750 & 1.000 & 0.833 & 1.000 & 0.917 & 0.933 \\
\hline 77 & - & - & - & - & - & - & - & - & - & - & 0.250 & - & 0.167 & - & - & 0.067 \\
\hline$M E^{*}$ & & & & & & & & & & & & & & & & \\
\hline$(\mathrm{N})$ & (7) & (6) & (5) & (6) & (11) & (6) & (7) & (9) & (5) & (5) & (14) & (7) & (3) & (6) & (6) & (15) \\
\hline 122 & - & - & - & 0.083 & - & - & - & 0.056 & 0.300 & - & - & - & - & - & - & - \\
\hline 100 & 1.000 & 1.000 & 1.000 & 0.917 & 1.000 & 1.000 & 0.929 & 0.833 & 0.700 & 0.900 & 0.893 & 0.857 & 0.833 & 0.667 & 1.000 & 0.733 \\
\hline 92 & - & - & - & - & - & - & 0.071 & 0.111 & - & 0.100 & 0.107 & 0.143 & 0.167 & 0.333 & - & 0.267 \\
\hline$P G M^{*}$ & & & & & & & & & & & & & & & & \\
\hline$(\mathrm{N})$ & (7) & (6) & (5) & (6) & (11) & (6) & (7) & (9) & (5) & (5) & (14) & (7) & (3) & (6) & (6) & (15) \\
\hline 135 & - & - & - & - & - & - & - & - & - & - & - & 0.143 & - & - & - & - \\
\hline 122 & - & - & - & - & - & 0.167 & - & - & - & 0.100 & - & - & 0.167 & - & - & 0.033 \\
\hline 111 & 0.357 & 0.083 & 0.200 & 0.083 & 0.045 & 0.083 & - & - & - & 0.500 & 0.143 & 0.286 & - & 0.333 & 0.417 & 0.133 \\
\hline 104 & 0.071 & 0.083 & 0.100 & 0.083 & 0.045 & - & - & 0.389 & 0.100 & 0.200 & 0.036 & 0.143 & 0.333 & 0.250 & 0.167 & 0.267 \\
\hline 100 & 0.429 & 0.833 & 0.700 & 0.833 & 0.864 & 0.750 & 1.000 & 0.611 & 0.900 & 0.200 & 0.714 & 0.429 & 0.500 & 0.417 & 0.333 & 0.533 \\
\hline 80 & 0.143 & - & - & - & 0.045 & - & - & - & - & - & 0.107 & - & - & - & 0.083 & - \\
\hline 63 & - & - & - & - & - & - & - & - & - & - & - & - & - & - & - & 0.033 \\
\hline
\end{tabular}

$E N O^{*}, L G G-2^{*}, L T-1^{*}$ and $L T-2^{*}$ were monomorphic for the alleles $E N O^{*} 100, L G G-2^{*} 100, L T-1^{*} 100$ and $L T-1^{*} 100$ respectively 
Table 3b. Gene frequencies at 11 allozyme loci for 15 populations in three morphological groupings

\begin{tabular}{|c|c|c|c|c|c|c|c|c|c|c|c|c|c|c|c|}
\hline \multirow[b]{2}{*}{$\begin{array}{l}\text { Locus/ } \\
\text { Allele }\end{array}$} & \multicolumn{5}{|c|}{ Protopalythoa mutuki } & \multicolumn{9}{|c|}{ Pro. form 2} & \multirow{2}{*}{$\begin{array}{l}\text { Pro. form } 3 \\
\text { Ross }\end{array}$} \\
\hline & Border & Heron & Low Is. & Mabuiag & $\begin{array}{l}\text { Towns- } \\
\text { ville }\end{array}$ & Border & $\begin{array}{l}\text { Endeav- } \\
\text { our }\end{array}$ & Orpheus & Goods & Heron & E. Hope & Low Is. & $21-299$ & Ross & \\
\hline \multicolumn{16}{|l|}{ ENO* } \\
\hline 136 & - & - & - & - & - & - & - & 0.019 & - & - & 0.050 & - & - & - & - \\
\hline 118 & - & 0.083 & 0.417 & - & 0.083 & 0.667 & 0.938 & 0.870 & 0.500 & 0.917 & 0.950 & 0.850 & 0.786 & 0.786 & - \\
\hline 100 & 1.000 & 0.917 & 0.583 & 1.000 & 0.917 & 0.333 & 0.063 & 0.111 & 0.500 & 0.083 & - & 0.150 & 0.214 & 0.214 & 1.000 \\
\hline \multicolumn{16}{|c|}{$E S T-D^{*}$} \\
\hline$(\mathrm{N})$ & (10) & (6) & (6) & (9) & (6) & (9) & (7) & (28) & (14) & (6) & (10) & (10) & (7) & (7) & (2) \\
\hline 114 & - & - & - & - & - & - & 0.071 & - & - & 0.083 & - & - & 0.071 & - & - \\
\hline 100 & 1.000 & 0.333 & 0.500 & 0.778 & 1.000 & 0.833 & 0.714 & 0.571 & 1.000 & 0.417 & 0.550 & 0.750 & 0.500 & 0.643 & 1.000 \\
\hline 72 & - & 0.667 & 0.500 & 0.056 & - & 0.056 & 0.214 & 0.393 & - & 0.500 & 0.450 & 0.250 & 0.429 & 0.357 & - \\
\hline 57 & - & - & - & - & - & 0.111 & - & 0.036 & - & - & - & - & - & - & - \\
\hline 51 & - & - & - & 0.167 & - & - & - & - & - & - & - & - & - & - & - \\
\hline $\begin{array}{l}G P^{*} \\
(\mathrm{~N})\end{array}$ & (10) & (6) & (6) & (9) & (6) & (9) & (8) & (28) & (14) & (6) & (10) & (10) & (7) & (7) & (2) \\
\hline 129 & - & 0.417 & 0.500 & 0.111 & - & - & - & - & - & - & - & - & - & - & - \\
\hline 111 & 0.500 & 0.417 & 0.500 & - & - & - & - & - & - & - & - & - & - & - & - \\
\hline 100 & 0.500 & 0.167 & - & 0.667 & 0.500 & - & - & - & - & - & - & - & - & - & - \\
\hline 81 & - & - & - & 0.222 & 0.500 & - & - & - & - & - & - & - & - & - & 1.000 \\
\hline 30 & - & - & - & - & - & 0.056 & - & - & - & - & - & 0.150 & - & - & - \\
\hline 22 & - & - & - & - & - & - & - & - & - & - & - & 0.050 & - & - & - \\
\hline 15 & - & - & - & - & - & 0.167 & 0.125 & 0.143 & - & 0.083 & 0.250 & 0.200 & - & 0.143 & - \\
\hline 0 & - & - & - & - & - & 0.722 & 0.750 & 0.625 & 1.000 & 0.833 & 0.450 & 0.450 & 0.714 & 0.643 & - \\
\hline$(-15)$ & - & - & - & - & - & 0.056 & 0.125 & 0.232 & - & 0.083 & 0.300 & 0.150 & 0.286 & 0.214 & - \\
\hline \multicolumn{16}{|l|}{$H K^{*}$} \\
\hline 97 & - & - & - & - & - & 0.063 & 0.125 & 0.125 & - & - & 0.150 & 0.050 & 0.143 & - & - \\
\hline 93 & 1.000 & 0.250 & 0.500 & 0.278 & - & - & - & - & - & - & - & - & - & - & - \\
\hline 87 & - & 0.583 & - & 0.111 & - & 0.313 & - & 0.161 & 0.208 & - & 0.050 & 0.250 & 0.143 & - & - \\
\hline 84 & - & 0.167 & 0.500 & 0.611 & 1.000 & - & - & - & - & - & - & - & - & - & - \\
\hline 78 & - & - & - & - & - & 0.250 & 0.188 & 0.214 & 0.542 & 0.333 & 0.250 & 0.150 & 0.286 & 0.286 & 0.750 \\
\hline 73 & - & - & - & - & - & 0.125 & 0.063 & 0.196 & 0.208 & 0.250 & 0.250 & 0.250 & 0.286 & 0.286 & 0.250 \\
\hline 50 & - & - & - & - & - & 0.188 & 0.313 & 0.054 & 0.042 & - & 0.150 & - & - & 0.286 & - \\
\hline 40 & - & - & - & - & - & 0.063 & 0.313 & 0.250 & - & 0.417 & 0.150 & 0.300 & 0.143 & 0.143 & - \\
\hline \multicolumn{16}{|l|}{$I D H^{*}$} \\
\hline 110 & - & 0.167 & - & - & 0.083 & - & - & - & - & - & - & - & - & - & - \\
\hline 100 & - & 0.833 & 1.000 & - & 0.417 & 0.063 & - & 0.036 & - & - & - & - & - & - & 1.000 \\
\hline 90 & 1.000 & - & - & 0.500 & 0.333 & 0.813 & 1.000 & 0.964 & 0.750 & 1.000 & 1.000 & 1.000 & 0.929 & 0.929 & - \\
\hline 80 & - & - & - & 0.500 & 0.167 & 0.125 & - & - & 0.250 & - & - & - & 0.071 & 0.071 & - \\
\hline \multicolumn{16}{|l|}{$L G G-1^{*}$} \\
\hline$(\mathrm{N})$ & (10) & (6) & (6) & (9) & (6) & (9) & (8) & (28) & (14) & (5) & (10) & (10) & (7) & (7) & (2) \\
\hline 122 & - & - & - & 0.056 & - & - & - & - & - & - & - & - & - & - & - \\
\hline 112 & - & 0.500 & 0.500 & 0.167 & - & 0.056 & - & 0.054 & 0.071 & - & - & 0.100 & 0.143 & 0.214 & - \\
\hline 102 & - & - & - & 0.167 & - & 0.500 & 0.938 & 0.589 & 0.357 & 0.800 & 0.950 & 0.550 & 0.357 & 0.500 & 0.500 \\
\hline 100 & 1.000 & 0.500 & 0.500 & 0.611 & 1.000 & 0.444 & 0.063 & 0.268 & 0.536 & 0.200 & 0.050 & 0.350 & 0.500 & 0.143 & 0.500 \\
\hline 94 & - & - & - & - & - & - & - & 0.089 & 0.036 & - & - & - & - & 0.143 & - \\
\hline \multicolumn{16}{|l|}{$L F^{*}$} \\
\hline$(\mathrm{N})$ & (10) & (6) & (6) & (9) & (6) & (9) & (8) & (28) & (14) & (6) & (10) & (10) & (7) & (7) & (2) \\
\hline 100 & 1.000 & 1.000 & 1.000 & 1.000 & 1.000 & 1.000 & 1.000 & 1.000 & 1.000 & 1.000 & 1.000 & 1.000 & 1.000 & 1.000 & 1.000 \\
\hline$M D H-1$ & & & & & & & & & & & & & & & \\
\hline$(\mathrm{N})$ & (10) & (6) & (6) & (8) & (6) & (9) & (8) & (28) & (14) & (6) & (10) & (10) & (7) & (7) & (2) \\
\hline 184 & - & - & - & - & - & - & - & - & - & - & - & - & - & - & 0.250 \\
\hline 176 & - & - & - & - & - & - & - & 0.036 & - & - & - & - & - & - & - \\
\hline 149 & - & - & - & - & - & 0.333 & 0.500 & 0.571 & - & 0.583 & 0.450 & 0.300 & 0.357 & 0.500 & - \\
\hline 141 & - & - & 0.500 & 0.125 & 0.500 & - & - & - & - & - & - & - & - & - & - \\
\hline 116 & - & - & - & 0.063 & - & 0.056 & - & - & - & - & - & - & - & - & - \\
\hline 100 & 1.000 & 1.000 & 0.500 & 0.813 & 0.500 & - & - & - & - & - & - & - & - & - & 0.750 \\
\hline 92 & - & - & - & - & - & 0.611 & 0.438 & 0.357 & 1.000 & 0.417 & 0.550 & 0.650 & 0.643 & 0.500 & - \\
\hline 68 & - & - & - & - & - & - & 0.063 & 0.036 & - & - & - & 0.050 & - & - & - \\
\hline$M D H-2$ & & & & & & & & & & & & & & & \\
\hline$(\mathrm{N})$ & (10) & $(6)$ & (6) & (9) & (6) & (6) & (8) & (14) & (14) & (6) & (10) & (10) & (7) & (7) & (2) \\
\hline 91 & - & - & 0.417 & 0.167 & 0.500 & - & - & - & - & - & - & - & - & - & - \\
\hline 86 & - & - & - & - & - & - & - & - & 0.286 & - & - & - & - & - & - \\
\hline
\end{tabular}


Table 3b. (Continued)

\begin{tabular}{|c|c|c|c|c|c|c|c|c|c|c|c|c|c|c|c|}
\hline \multirow[b]{2}{*}{$\begin{array}{l}\text { Locus/ } \\
\text { Allele }\end{array}$} & \multicolumn{5}{|c|}{ Protopalythoa mutuki } & \multicolumn{9}{|c|}{ Pro. form 2} & \multirow{2}{*}{$\frac{\text { Pro. form } 3}{\text { Ross }}$} \\
\hline & Border & Heron & Low Is. & Mabuiag & $\begin{array}{l}\text { Towns- } \\
\text { ville }\end{array}$ & Border & $\begin{array}{l}\text { Endeav- } \\
\text { our }\end{array}$ & Orpheus & Goods & Heron & E. Hope & Low Is. & $21-299$ & Ross & \\
\hline 77 & 1.000 & 1.000 & 0.583 & 0.833 & 0.500 & 0.833 & 0.813 & 0.786 & 0.714 & 0.667 & 0.700 & 0.800 & 0.571 & 0.786 & 1.000 \\
\hline 59 & - & - & - & - & - & 0.083 & - & 0.036 & - & - & - & 0.150 & 0.286 & - & - \\
\hline 32 & - & - & - & - & - & 0.083 & 0.188 & 0.143 & - & 0.333 & 0.250 & 0.050 & 0.143 & 0.214 & - \\
\hline 14 & - & - & - & - & - & - & - & 0.036 & - & - & - & - & - & - & - \\
\hline 10 & - & - & - & - & - & - & - & - & - & - & 0.050 & - & - & - & - \\
\hline $\begin{array}{l}M E^{*} \\
(\mathrm{~N})\end{array}$ & & & & & & & & & & & & & & & \\
\hline $\begin{array}{l}\text { (N) } \\
122\end{array}$ & $\begin{array}{l}\text { (10) } \\
1.000\end{array}$ & $\begin{array}{l}\text { (0) } \\
0.750\end{array}$ & $\begin{array}{l}\text { (0) } \\
0.917\end{array}$ & 1.000 & $\begin{array}{l}\text { (b) } \\
1.000\end{array}$ & (8) & $\begin{array}{l}\text { (8) } \\
0.438\end{array}$ & $\begin{array}{l}(28) \\
0.304\end{array}$ & $\begin{array}{l}(13) \\
0.192\end{array}$ & $\begin{array}{l}\text { (6) } \\
-\end{array}$ & $\begin{array}{l}(10) \\
0.250\end{array}$ & (10) & (7) & (7) & (2) \\
\hline 100 & - & - & 0.083 & - & - & 0.250 & 0.125 & 0.268 & 0.731 & - & 0.250 & 0.250 & 0.286 & 0.143 & - \\
\hline 94 & - & - & - & - & - & - & - & - & - & - & - & - & - & - & 1.000 \\
\hline 92 & - & 0.250 & - & - & - & 0.750 & 0.438 & 0.429 & 0.077 & 1.000 & 0.500 & 0.750 & 0.714 & 0.643 & - \\
\hline 86 & - & - & - & - & - & - & - & - & - & - & - & - & - & 0.214 & - \\
\hline$P G M^{*}$ & & & & & & & & & & & & & & & \\
\hline & (10) & (6) & (6) & (9) & (6) & (8) & (8) & (28) & (12) & (6) & (9) & (10) & (7) & (7) & (2) \\
\hline 122 & 0.500 & 0.167 & - & 0.278 & - & 0.125 & 0.063 & - & - & - & 0.111 & - & - & - & - \\
\hline 118 & - & - & 0.500 & - & - & - & - & - & - & - & - & - & - & - & - \\
\hline 111 & - & 0.167 & - & 0.111 & - & - & - & 0.018 & - & - & - & 0.050 & - & - & - \\
\hline 107 & - & 0.083 & 0.500 & 0.333 & 0.500 & 0.188 & 0.188 & 0.196 & 0.583 & 0.167 & 0.167 & 0.350 & 0.429 & 0.286 & 0.750 \\
\hline 103 & 0.500 & 0.333 & - & 0.167 & 0.500 & - & - & - & - & - & - & - & - & - & - \\
\hline 100 & - & 0.250 & - & 0.111 & - & 0.563 & 0.688 & 0.696 & 0.417 & 0.833 & 0.667 & 0.500 & 0.429 & 0.643 & 0.250 \\
\hline 80 & - & - & - & - & - & 0.125 & 0.063 & 0.089 & - & - & 0.056 & 0.100 & 0.143 & 0.071 & - \\
\hline
\end{tabular}

$L G G-2^{*}, L T-1^{*}$ and $L T-2^{*}$ were monomorphic for the alleles $L G G-2^{*} 100 L T-1^{*} 100$ and $L T-2 * 100$ respectively

Table 3c. Gene frequencies at 13 enzyme loci for 14 populations of six morphological groupings

\begin{tabular}{|c|c|c|c|c|c|c|c|c|c|c|c|c|c|c|}
\hline \multirow[b]{2}{*}{$\begin{array}{l}\text { Locus/ } \\
\text { Allele }\end{array}$} & \multicolumn{3}{|c|}{ Sphenopus marsupialis } & \multicolumn{4}{|c|}{ Zoanthus coppingeri } & \multicolumn{2}{|c|}{$Z$. form 2} & \multicolumn{3}{|c|}{$Z$. form 3} & \multirow{2}{*}{$\begin{array}{l}Z \text {. } \\
\text { vietnamensis } \\
\text { Heron }\end{array}$} & \multirow{2}{*}{$\begin{array}{l}\begin{array}{l}\text { Parazoanthus } \\
\text { dichroicus }\end{array} \\
\text { Bowden }\end{array}$} \\
\hline & $\begin{array}{l}\text { Cleve- } \\
\text { land }\end{array}$ & $\begin{array}{l}\text { Mag- } \\
\text { netic }\end{array}$ & $\begin{array}{l}\text { Cape } \\
\text { York }\end{array}$ & Goods & Low Is. & Mabuiag & $\begin{array}{l}\text { Towns- } \\
\text { ville }\end{array}$ & Low Is. & $\begin{array}{l}\text { Towns- } \\
\text { ville }\end{array}$ & Heron & Low Is & . 21-299 & & \\
\hline $\begin{array}{l}E N O^{*} \\
(\mathrm{~N})\end{array}$ & (10) & (10) & (5) & (13) & (5) & (9) & (6) & (6) & (6) & (6) & (6) & (6) & (6) & (6) \\
\hline 118 & 0.450 & 0.100 & 0.100 & - & - & - & - & - & - & - & - & - & - & - \\
\hline 100 & 0.500 & 0.850 & 0.800 & - & - & - & - & - & - & - & - & - & 1.000 & - \\
\hline 82 & 0.050 & 0.050 & 0.100 & - & - & - & - & - & - & - & - & - & - & - \\
\hline 79 & - & - & - & 0.385 & 0.500 & 0.167 & 0.333 & 0.083 & 0.667 & 0.250 & 0.167 & - & - & 0.833 \\
\hline 68 & - & - & - & - & - & - & - & - & - & - & - & - & - & 0.167 \\
\hline 61 & - & - & - & 0.615 & 0.500 & 0.833 & 0.500 & 0.917 & 0.333 & 0.667 & 0.833 & 1.000 & - & - \\
\hline 57 & - & - & - & - & - & - & - & - & - & 0.083 & - & - & - & - \\
\hline 43 & - & - & - & - & - & - & 0.167 & - & - & - & - & - & - & - \\
\hline \multicolumn{15}{|l|}{$E S T-D^{*}$} \\
\hline 125 & 0.050 & - & - (1) & - & 0.200 & - & 0.083 & 0.083 & - & 0.083 & - & - & (1) & - \\
\hline 100 & 0.900 & 0.750 & 0.900 & 0.423 & 0.800 & 0.778 & 0.917 & 0.667 & 0.833 & 0.917 & 0.833 & 1.000 & 0.583 & - \\
\hline 72 & 0.050 & 0.250 & 0.100 & 0.577 & - & 0.222 & - & 0.250 & 0.167 & - & 0.167 & - & 0.417 & - \\
\hline \multicolumn{15}{|l|}{$G P^{*}$} \\
\hline$(\mathrm{N})$ & (10) & (10) & (5) & (13) & (5) & (9) & (6) & (6) & (6) & (6) & (6) & (6) & (6) & (6) \\
\hline 111 & - & - & - & - & - & 0.111 & 0.083 & - & 0.333 & - & - & - & - & - \\
\hline 104 & - & - & - & 0.385 & - & 0.167 & - & - & - & - & 0.083 & - & - & - \\
\hline 86 & - & - & - & - & - & - & 0.083 & - & 0.167 & - & 0.083 & - & - & - \\
\hline 78 & - & - & - & 0.077 & 0.200 & 0.333 & - & 0.083 & - & - & 0.333 & - & - & 0.083 \\
\hline 67 & - & - & - & 0.077 & 0.200 & 0.278 & $0 . .417$ & 0.500 & 0.500 & 0.583 & 0.167 & 0.833 & - & 0.583 \\
\hline 53 & - & - & - & - & - & - & - & - & - & - & - & - & - & 0.167 \\
\hline 48 & 0.050 & 0.050 & - & 0.346 & 0.400 & 0.056 & 0.083 & 0.167 & - & 0.167 & 0.167 & 0.083 & - & - \\
\hline 44 & - & - & - & - & - & - & 0.083 & - & - & - & - & - & 0.083 & 0.167 \\
\hline 37 & 0.950 & 0.950 & 1.000 & 0.115 & 0.200 & 0.056 & 0.250 & 0.250 & - & 0.250 & 0.167 & 0.083 & - & - \\
\hline 30 & - & - & - & - & - & - & - & - & - & - & - & - & 0.917 & - \\
\hline \multicolumn{15}{|l|}{$H K^{*}$} \\
\hline (N) & (10) & (7) & (5) & (1) & (1) & (1) & (6) & (6) & (6) & (6) & (5) & (6) & (6) & (6) \\
\hline 132 & 0.500 & 0.286 & 0.100 & - & - & - & - & - & - & - & - & - & - & - \\
\hline 130 & 0.200 & 0.286 & 0.600 & - & - & - & - & - & - & - & - & - & - & - \\
\hline
\end{tabular}


Table 3c. (Continued)

\begin{tabular}{|c|c|c|c|c|c|c|c|c|c|c|c|c|c|c|}
\hline \multirow[b]{2}{*}{$\begin{array}{l}\text { Locus/ } \\
\text { Allele }\end{array}$} & \multicolumn{3}{|c|}{ Sphenopus marsupialis } & \multicolumn{4}{|c|}{ Zoanthus coppingeri } & \multicolumn{2}{|c|}{$Z$. form 2} & \multicolumn{3}{|c|}{$Z$. form 3} & \multirow{2}{*}{$\frac{\begin{array}{l}Z . \\
\text { vietnamensis }\end{array}}{\text { Heron }}$} & \multirow{2}{*}{$\begin{array}{l}\begin{array}{l}\text { Parazoanthus } \\
\text { dichroicus }\end{array} \\
\text { Bowden }\end{array}$} \\
\hline & $\begin{array}{l}\text { Cleve- } \\
\text { land }\end{array}$ & $\begin{array}{l}\text { Mag- } \\
\text { netic }\end{array}$ & $\begin{array}{l}\text { Cape } \\
\text { York }\end{array}$ & Goods & Low Is. & Mabuiag & $\begin{array}{l}\text { Towns- } \\
\text { ville }\end{array}$ & Low Is. & $\begin{array}{l}\text { Towns- } \\
\text { ville }\end{array}$ & Heron & Low Is & 21-299 & & \\
\hline 128 & 0.250 & 0.357 & 0.100 & - & - & - & - & - & - & - & - & - & - & - \\
\hline 124 & 0.050 & 0.071 & 0.200 & - & - & - & - & - & - & - & - & - & - & - \\
\hline 110 & - & - & - & - & - & - & - & - & - & - & - & - & - & 0.083 \\
\hline 100 & - & - & - & - & - & - & - & - & - & - & - & - & - & 0.417 \\
\hline 87 & - & - & - & - & - & - & - & - & - & - & - & - & 0.667 & 0.417 \\
\hline 84 & - & - & - & - & - & - & - & 0.083 & - & - & - & 0.083 & 0.083 & - \\
\hline 78 & - & - & - & 1.000 & 1.000 & 1.000 & 0.583 & 0.833 & 0.333 & 0.167 & 0.500 & - & 0.250 & - \\
\hline 73 & - & - & - & - & - & - & - & - & - & - & - & - & - & 0.083 \\
\hline 50 & - & - & - & - & - & - & 0.417 & 0.083 & 0.667 & 0.833 & 0.500 & 0.917 & - & - \\
\hline \multicolumn{15}{|l|}{$L G G-1 *$} \\
\hline $\begin{array}{l}\text { (N) } \\
122\end{array}$ & $\begin{array}{l}(10) \\
0.850\end{array}$ & $\begin{array}{l}(7) \\
0.929\end{array}$ & $\begin{array}{l}(5) \\
1.000\end{array}$ & $\begin{array}{l}(13) \\
-\end{array}$ & (5) & $\begin{array}{l}\text { (9) } \\
-\end{array}$ & $\begin{array}{l}\text { (6) } \\
-\end{array}$ & (6) & $\begin{array}{l}(6) \\
-\end{array}$ & $\begin{array}{l}(6) \\
-\end{array}$ & $\begin{array}{l}(6) \\
-\end{array}$ & $\begin{array}{l}(6) \\
-\end{array}$ & $\begin{array}{l}(6) \\
-\end{array}$ & $\begin{array}{l}(6) \\
-\end{array}$ \\
\hline 118 & 0.150 & 0.071 & - & - & - & - & - & - & - & - & - & - & - & - \\
\hline 114 & - & - & - & 0.346 & 0.400 & 0.556 & 0.500 & 0.583 & 0.667 & 0.750 & 0.500 & 0.917 & - & - \\
\hline 106 & - & - & - & - & - & - & - & - & 0.167 & - & - & - & - & - \\
\hline 100 & - & - & - & 0.654 & 0.600 & 0.444 & 0.500 & 0.417 & 0.167 & 0.250 & 0.500 & 0.083 & 0.500 & 1.000 \\
\hline 84 & - & - & - & - & - & - & - & - & - & - & - & - & 0.500 & - \\
\hline \multicolumn{15}{|l|}{$L G G-2^{*}$} \\
\hline 104 & (10) & (I0) & (ग) & (ID) & (J) & (9) & (0) & (0) & $\begin{array}{l}\text { (0) } \\
-\end{array}$ & $\begin{array}{l}\text { (0) } \\
-\end{array}$ & (6) & $\begin{array}{l}\text { (0) } \\
-\end{array}$ & 1.000 & (0) \\
\hline 100 & 1.000 & 1.000 & 1.000 & 1.000 & 1.000 & 1.000 & 1.000 & 1.000 & 1.000 & 1.000 & 1.000 & 1.000 & - & - \\
\hline \multicolumn{15}{|l|}{$L P^{*}$} \\
\hline 114 & $\begin{array}{l}\text { (10) } \\
0.500\end{array}$ & $\begin{array}{l}\text { (10) } \\
0.700\end{array}$ & $\begin{array}{l}0.800 \\
0.07\end{array}$ & $\begin{array}{l}\text { (13) } \\
-\end{array}$ & (3) & $\begin{array}{l}\text { (9) } \\
-\end{array}$ & (b) & $\begin{array}{l}\text { (6) } \\
-\end{array}$ & $\begin{array}{l}\text { (6) } \\
-\end{array}$ & $\begin{array}{l}\text { (6) } \\
-\end{array}$ & $\begin{array}{l}\text { (6) } \\
-\end{array}$ & $\begin{array}{l}\text { (b) } \\
-\end{array}$ & $\begin{array}{l}\text { (b) } \\
-\end{array}$ & 0.250 \\
\hline 108 & 0.500 & 0.300 & 0.200 & - & - & - & - & - & - & - & - & - & - & - \\
\hline 104 & - & - & - & - & - & - & - & - & - & - & - & - & 1.000 & - \\
\hline 100 & - & - & - & 1.000 & 1.000 & 1.000 & 1.000 & 1.000 & 1.000 & 1.000 & 1.000 & 1.000 & - & 0.750 \\
\hline \multicolumn{15}{|l|}{$L T-1^{*}$} \\
\hline 100 & 1.000 & 1.000 & 1.000 & 1.000 & 1.000 & 1.000 & 1.000 & 1.000 & 1.000 & 1.000 & $\begin{array}{l}\text { (d) } \\
1.000\end{array}$ & 1.000 & $\begin{array}{l}\text { (0) } \\
-\end{array}$ & $\begin{array}{l}\text { (0) } \\
-\end{array}$ \\
\hline 96 & - & - & - & - & - & - & - & - & - & - & - & - & - & 1.000 \\
\hline 94 & - & - & - & - & - & - & - & - & - & - & - & - & 1.000 & - \\
\hline \multicolumn{15}{|l|}{$L T-2^{*}$} \\
\hline (N) & (10) & (10) & (5) & (13) & (5) & (9) & (6) & (6) & (6) & (6) & (6) & (6) & (6) & (6) \\
\hline 103 & - & - & - & - & - & - & - & - & - & - & - & - & - & 1.000 \\
\hline 100 & 1.000 & 1.000 & 1.000 & 1.000 & 1.000 & 1.000 & 1.000 & 1.000 & 1.000 & 1.000 & 1.000 & 1.000 & - & - \\
\hline 66 & - & - & - & - & - & - & - & - & - & - & - & - & 1.000 & - \\
\hline \multicolumn{15}{|l|}{$M D H-1^{*}$} \\
\hline & (10) & (7) & (5) & (13) & (5) & (9) & (6) & (6) & (6) & (5) & (6) & (6) & (6) & (6) \\
\hline 149 & - & - & - & - & - & - & - & - & - & - & - & - & - & 0.083 \\
\hline 96 & 0.050 & 0.071 & 0.100 & - & - & - & - & - & - & - & - & - & - & - \\
\hline 92 & 0.950 & 0.857 & 0.900 & - & - & - & - & - & - & - & - & - & - & - \\
\hline 76 & - & - & - & - & - & - & 0.250 & - & 0.167 & 0.100 & - & 0.417 & - & - \\
\hline 68 & - & 0.071 & - & - & - & - & - & - & - & - & 0.083 & - & - & - \\
\hline 62 & - & - & - & 0.346 & - & 0.667 & 0.500 & 0.417 & 0.667 & 0.400 & 0.583 & 0.167 & - & 0.417 \\
\hline 59 & - & - & - & - & - & - & - & - & - & - & - & - & 1.000 & - \\
\hline 57 & - & - & - & - & - & - & - & - & - & - & 0.083 & - & - & - \\
\hline 51 & - & - & - & - & - & - & - & - & - & - & - & - & - & 0.500 \\
\hline 49 & - & - & - & 0.654 & 1.000 & 0.333 & 0.250 & 0.583 & 0.167 & 0.500 & 0.250 & 0.417 & - & - \\
\hline $\begin{array}{l}M D H-2^{*} \\
(\mathrm{~N})\end{array}$ & (10) & (7) & (5) & (13) & (5) & (9) & (6) & (6) & (6) & (6) & (6) & (6) & (6) & (6) \\
\hline 136 & - & - & - & 0.038 & 0.200 & 0.056 & - & - & - & - & - & - & - & - \\
\hline 118 & - & - & - & - & - & 0.056 & - & - & 0.167 & - & - & - & - & - \\
\hline 105 & 0.250 & 0.071 & - & - & - & - & - & - & - & - & - & - & - & - \\
\hline 86 & 0.700 & 0.500 & 0.700 & - & - & - & - & - & - & - & - & - & - & - \\
\hline 77 & 0.050 & 0.429 & 0.300 & - & - & - & - & - & - & - & - & - & 0.083 & - \\
\hline 68 & - & - & - & 0.962 & 0.800 & 0.889 & 0.917 & 1.000 & 0.833 & 1.000 & 1.000 & 1.000 & - & - \\
\hline 55 & - & - & - & - & - & - & - & - & - & - & - & - & 0.667 & - \\
\hline 45 & - & - & - & - & - & - & - & - & - & - & - & - & - & 1.000 \\
\hline 36 & - & - & - & - & - & - & 0.083 & - & - & - & - & - & - & - \\
\hline 23 & - & - & - & - & - & - & - & - & - & - & - & - & 0.250 & - \\
\hline
\end{tabular}


Table 3c. (Continued)

\begin{tabular}{|c|c|c|c|c|c|c|c|c|c|c|c|c|c|c|}
\hline \multirow[b]{2}{*}{$\begin{array}{l}\text { Locus/ } \\
\text { Allele }\end{array}$} & \multicolumn{3}{|c|}{ Sphenopus marsupialis } & \multicolumn{4}{|c|}{ Zoanthus coppingeri } & \multicolumn{2}{|c|}{$Z$. form 2} & \multicolumn{3}{|c|}{$Z$. form 3} & \multirow{2}{*}{$\begin{array}{l}Z . \\
\text { vietnamensis } \\
\text { Heron }\end{array}$} & \multirow{2}{*}{$\begin{array}{l}\text { Parazoanthus } \\
\text { dichroicus }\end{array}$} \\
\hline & $\begin{array}{l}\text { Cleve- } \\
\text { land }\end{array}$ & $\begin{array}{l}\text { Mag- } \\
\text { netic }\end{array}$ & $\begin{array}{l}\text { Cape } \\
\text { York }\end{array}$ & Goods & Low Is. & Mabuiag & $\begin{array}{l}\text { Towns- } \\
\text { ville }\end{array}$ & Low Is. & $\begin{array}{l}\text { Towns- } \\
\text { ville }\end{array}$ & Heron & Low I & Is. $21-299$ & & \\
\hline \multicolumn{15}{|l|}{$M E^{*}$} \\
\hline $\begin{array}{l}\text { (N) } \\
126\end{array}$ & $\begin{array}{l}(10) \\
-\end{array}$ & $\begin{array}{ll}(10) \\
-\end{array}$ & $\begin{array}{l}\text { (ग) } \\
-\end{array}$ & $\begin{array}{l}(12) \\
-\end{array}$ & $\begin{array}{l}(4) \\
-\end{array}$ & $\begin{array}{l}\text { (8) } \\
-\end{array}$ & $\begin{array}{l}\text { (b) } \\
0.250\end{array}$ & $\begin{array}{l}\text { (J) } \\
-\end{array}$ & $\begin{array}{l}\text { (b) } \\
-\end{array}$ & $\begin{array}{l}\text { (b) } \\
0.083\end{array}$ & $\begin{array}{l}\text { (b) } \\
-\end{array}$ & $\begin{array}{l}\text { (b) } \\
0.333\end{array}$ & $\begin{array}{l}\text { (b) } \\
0.167\end{array}$ & $\begin{array}{l}\text { (b) } \\
-\end{array}$ \\
\hline 122 & 0.050 & 0.050 & - & - & - & - & - & - & - & - & - & - & - & - \\
\hline 110 & - & - & - & 0.208 & - & 0.250 & 0.250 & 0.600 & 0.417 & 0.167 & 0.500 & - & - & - \\
\hline 100 & 0.950 & 0.950 & 1.000 & 0.250 & 1.000 & 0.563 & - & 0.100 & - & 0.500 & 0.333 & 0.667 & 0.417 & - \\
\hline 92 & - & - & - & 0.542 & - & 0.188 & 0.500 & 0.300 & 0.500 & 0.167 & - & - & 0.250 & - \\
\hline 88 & - & - & - & - & - & - & - & - & 0.083 & 0.083 & 0.167 & - & 0.167 & - \\
\hline \multicolumn{15}{|l|}{$P G M^{*}$} \\
\hline$(\mathrm{N})$ & (10) & (7) & (5) & (13) & (5) & (9) & (6) & (6) & (6) & (6) & (3) & (5) & (6) & (6) \\
\hline 111 & - & 0.143 & - & - & - & - & 0.083 & - & 0.167 & - & 0.333 & - & - & - \\
\hline 109 & - & - & - & 0.115 & 0.200 & 0.056 & - & 0.250 & - & - & - & 0.200 & - & - \\
\hline 104 & 0.450 & 0.071 & 0.700 & - & - & - & 0.417 & - & 0.167 & - & 0.333 & 0.200 & - & - \\
\hline 103 & - & - & - & 0.423 & 0.200 & 0.333 & 0.083 & 0.500 & - & 0.167 & 0.167 & - & - & - \\
\hline 102 & 0.450 & 0.786 & 0.300 & - & - & 0.056 & 0.167 & - & 0.167 & - & - & 0.400 & - & - \\
\hline 100 & - & - & - & 0.346 & 0.600 & 0.389 & 0.250 & 0.167 & 0.333 & 0.667 & 0.167 & 0.200 & 0.417 & - \\
\hline 90 & 0.100 & - & - & - & - & - & - & - & - & - & - & - & - & - \\
\hline 80 & - & - & - & - & - & - & - & - & - & - & - & - & 0.583 & - \\
\hline 55 & - & - & - & 0.115 & - & 0.167 & - & 0.083 & 0.167 & 0.167 & - & - & - & - \\
\hline 50 & - & - & - & - & - & - & - & - & - & - & - & - & - & 1.000 \\
\hline
\end{tabular}

$I D H^{*}$ was monomorphic for the allele $I D H^{*} 80$

from sympatric samples were combined, there were no significant deviations from Hardy-Weinberg equilibrium with the exception of ENO* from Kissing Point which shows a significant deficit of $* 79 / * 61$ heterozygotes (expected 5.2, observed 0 ). In contrast, $Z$. vietnamensis shows fixed gene differences from all other Zoanthus samples (including sympatric Heron I. $Z$. form 3 ) at seven loci $\left(E N O^{*}, L G G-2^{*}, L P^{*}, L T-1^{*}, L T-2^{*}, M D H-1^{*}, M D H-2^{*}\right)$ and $\mathrm{D}$ is correspondingly high $(>1.4$ in all comparisons of $Z$. vietnamensis with other Zoanthus forms). Our samples are considered to represent two species, identified as $Z$. coppingeri Haddon and Shackleton, 1891 and Z. vietnamensis Pax and Muller, 1957. The large differences seen among these two species suggest they may not be congeneric if genetic data are considered in isolation.

While genetic distances were relatively small within groups, some genetic heterogeneity was evident among samples of all genetic groups. This may reflect intraspecific genetic structure within the GBR region which has previously been observed in Palythoa caesia (Burnett et al. 1994) and Zoanthus coppingeri (Burnett et al. 1995). The effect may have been exaggerated by sampling errors due to our small sample sizes. Data for each group from all locations were pooled prior to phylogenetic analysis in order to give a conservative estimate of gene frequencies throughout the region of study. Pooled data for all samples of each species are highly variable (Table 4) with many loci being polymorphic and with high average heterozygosities. The large number of alleles detected meant there was a risk that some rare alleles shared between species may have represented stochastic convergence of electromorphs. In phylogenetic analyses, common alleles were considered more likely to be evolutionarily significant or informative than rare alleles. Additionally, the small sample sizes necessitated by the broad scope of the study introduced the risk that rare alleles were not sampled in some populations. This can affect phylogenetic reconstructions when polymorphic synapomorphies are common (Hillis 1987). To minimise the effect of these two problems, alleles with frequencies of less than 0.1 were combined with the most common allele in each locus for each species prior to phylogenetic analysis.

The phylogeny estimated from maximum likelihood analysis is shown in Fig. 3. Relative positions of Palythoa caesia, Protopalythoa mutuki and Pro. sp 3 are highly variable among the set of bootstrapped trees. The positions of Zoanthus coppingeri, $Z$. vietnamensis and Sphenopus marsupialis are more robust, but still show some flexibility. Overall bootstrap support for the phylogeny is low, however the tree is well supported by the available morphological data which is discussed in more detail later. The tree confirms the monophyly of the Palythoa/Protopalythoa group and their close relationship with Sphenopus as proposed by Haddon and Shackleton (1891). Zoanthus appears to be paraphyletic.

\section{Discussion}

\section{Species on the Great Barrier Reef}

Genetic evidence points to the existence of a relatively small number of species of GBR Zoanthidae. Only seven were detected in the samples collected, identified as Palythoa caesia, Protopalythoa mutuki, Pro. sp. 2, Pro. sp. 3, Sphenopus marsupialis, Zoanthus coppingeri and 


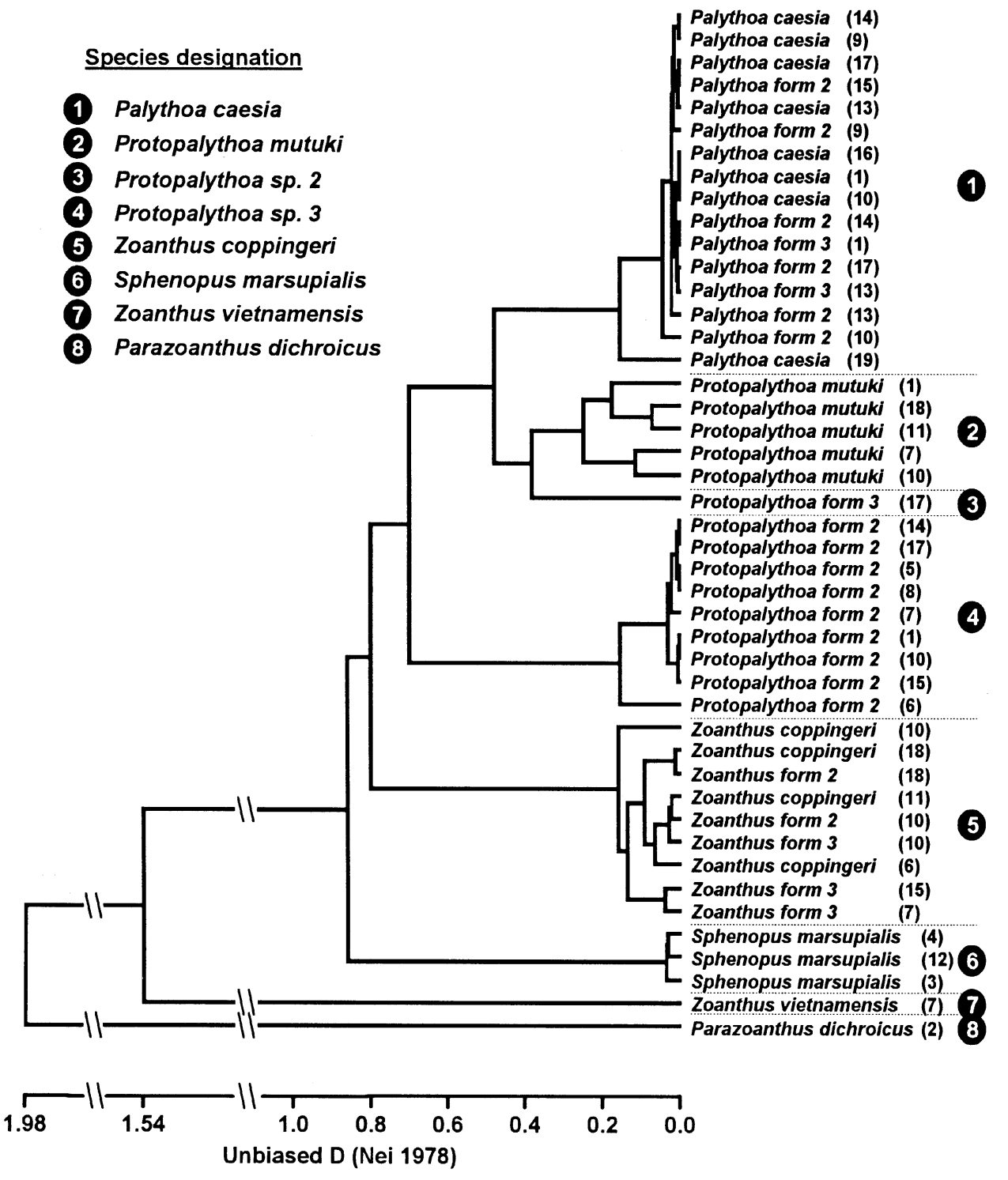

Key to localities:

1: Border I.; 2: Bowden Reef; 3: Cape Cleveland; 4: Cape York; 5: Endeavour Reef; 6: Goods I.;

7: Heron I.; 8: East Hope I.; 9: Hyde Recf; 10: Low Is.; 11: Mabuiag I.; 12: Magnetic I.; 13:

Myrmidon Reef; 14: Orpheus I; 15: Reef 21-299; 16: Reef 21-491; 17: Ross Reef; 18: Townsville;

19: Tuesday Is.

Fig. 2. UPGMA cluster analysis, using Nei's (1978) unbiased D, of 45 samples of Zoanthidae. Figures in brackets denote sample locality

Table 4. Genetic variability measures in eight species of Zoanthidea. Standard errors in parentheses. A locus was considered polymorphic if the most common allele was present at a frequency less than $99 \% . H_{0}$ : observed heterozygosity; $H_{e}$ : expected heterozygosity (unbiased estimate, Nei 1978)

\begin{tabular}{|c|c|c|c|c|c|}
\hline Species & $\begin{array}{l}\text { Mean sample } \\
\text { size per locus }\end{array}$ & $\begin{array}{l}\text { Mean alleles } \\
\text { per locus }\end{array}$ & $\begin{array}{l}\% \text { loci } \\
\text { polymorphic }\end{array}$ & $H_{\mathrm{o}}$ & $H_{e}$ \\
\hline Palythoa caesia & $117.6(0.3)$ & $3.5(0.6)$ & 71.4 & $0.271(0.071)$ & $0.285(0.068)$ \\
\hline Protopalythoa mutuki & $36.9(0.1)$ & $2.7(0.4)$ & 71.4 & $0.285(0.078)$ & $0.320(0.076)$ \\
\hline Protopalythoa sp. 2 & $96.8(1.2)$ & $3.5(0.5)$ & 71.4 & $0.298(0.066)$ & $0.350(0.073)$ \\
\hline Protopalythoa sp. 3 & $2 \quad(0)$ & $1.3(0.1)$ & 28.6 & $0.179(0.085)$ & $0.155(0.069)$ \\
\hline Zoanthus coppingeri & $60.4(2.0)$ & $3.4(0.6)$ & 64.3 & $0.322(0.085)$ & $0.350(0.085)$ \\
\hline Zoanthus vietnamensis & $6 \quad(0)$ & $1.8(0.3)$ & 50 & $0.310(0.102)$ & $0.258(0.078)$ \\
\hline Sphenopus marsupialus & $23.9(0.4)$ & $2.3(0.3)$ & 71.4 & $0.226(0.062)$ & $0.254(0.068)$ \\
\hline Parazoanthus dichroicus & $6 \quad(0)$ & $1.7(0.3)$ & 35.7 & $0.202(0.078)$ & $0.192(0.076)$ \\
\hline
\end{tabular}


$Z$. vietnamensis. This is substantially fewer than the $25+$ species described or previously reported from the region. We have identified five of the seven species described here from the literature. These five are considered to be probable senior synonyms of at least eighteen other nominate Australian species listed by Burnett (1995b). Pro. sp. 2 and Pro. sp. 3 could not be identified with any previously described species from Australia, although sp. 2 has been the subject of reproductive and developmental work by Babcock and Ryland (1990) and Ryland and Babcock (1991). These species may be described from elsewhere in the Indo-Pacific region.

The samples collected in this study include a wide range of morphologies from a variety of habitats. Our data suggest that not all morphological or ecological groupings represent evolutionarily independent taxa. The misinterpretation of this variability is probably responsible for the large number of described species found in the literature. This has already been shown to be the case for the zoanthid genus Isaurus (Muirhead and Ryland 1985). It appears likely that the number of zoanthid species found on the GBR, and probably worldwide, has been greatly exaggerated.

All three forms of Palythoa are genetically homogeneous and are considered a single species. It is possible, however, that a more powerful analysis may reveal genetic markers for the distinctive morphologies seen in this genus and cast more light on the intra- or interspecific nature of this morphological variation. Three of the four Zoanthus morphs examined also belong to a single species. The absence of $E N O^{*} 61 / * 79$ heterozygotes in Kissing Point Zoanthus coppingeri and $Z$. form 2 collections could represent a fixed difference between two cryptic species which do not correspond to either morphological form.
However, ENO*61/*79 heterozygotes are common in other samples, and were detected in larger collections from Kissing Point in a previous study (Burnett et al. 1995). Consequently the heterozygote deficit detected here may reflect sampling error. All Zoanthus samples except $Z$. vietnamensis are considered to be members of Z. coppingeri. Zoanthus coppingeri could potentially include several nominate species from Australia which were not identified in this study (Z. jukesii Haddon and Shackleton, 1891, Z. macgillivrayi H. \& S., 1891, Z. annae Carlgren 1937, Z. mantoni Carlgren 1937, Z. fraseri Carlgren 1937) but which are described from the same localities and which are indistinguishable in their external appearance from $Z$. coppingeri. These species were defined by Carlgren (1937) on the basis of differences in the structure and distribution of nematocysts. Such differences were considered by Muirhead (1989) not to be valid specific characters. Zoanthus coppingeri was described as Z. mantoni by Ryland and Muirhead (1993) and probably also includes $Z$. pacificus (Walsh and Bowers, 1971). In contrast, the status of Zoanthus vietnamensis is clear, as it shows fixed gene differences at seven loci in comparisons with other Zoanthus samples and is morphologically distinct.

The power of our analysis is limited by the number of loci surveyed and to some extent by sample sizes. The possibility of closely related sibling species being present within the seven species delimited here cannot be discounted. Low levels of genetic differentiation among closely related morphological species occurs in other cnidarians (e.g. Solé-Cava et al. 1985; Miller and Benzie in press). Fixed differences among incipient species may take a long time to arise, particularly if effective population sizes are large (Wright 1978). Large effective population sizes seem likely given that gene flow among GBR zoan-

Table 5. Key to Great Barrier Reef and Torres Strait Zoanthidae

1. A Polyps sand encrusted

B Polyps not sand encrusted

2. A Polyps solitary. Found living on sublittoral sandy substrata, not on coral reefs. Polyps very robust, rounded or slightly pointed at the base. Small specimens may be attached to small stones or sea grass blades by a thin stalk B Polyps colonial, on coral reefs and rocky shores

3. A Polyps completely immersed in a thick coenenchyme when closed. Colonies may be small $(\sim 3-10 \mathrm{~cm})$ and ovoid with large polyps, or very large with small, densely packed polyps

B Polyps free standing, connected only at base

4 A Intertidal. Disk green or brown, often with contrasting light coloured mouth and hypostome. Polyps cylindrical when closed, with scapus and capitulum of equal diameter. Scapus may appear transversely wrinkled B At exteme low water and sublittoral

Protopalythoa mutuki

5. A Polyps fully open in shade. Disk uniform brown, mottled white and brown/green, or brown with white radial lines. Often semi-closed in direct sunlight, with "triangular" appearance. Polyps trumpet shaped when closed Polyps white or sandy coloured, partially buried in coral rock

6. A Polyps large (to $70 \mathrm{~mm}$ ), joined at base. Colonies usually with only a few polyps. Body wall with large tubercules usually arranged in longitudinal series. Intertidal and sublittoral

B Polyps small (rarely over $20 \mathrm{~mm}$ ), wirhout tubercules or immersed in a thick coenenchyme

7. A Polyps joined at base by stolons. Intertidal or sublittoral. Colour highly variable, bright green, red, orange yellow or brown. Oral disk uniform or patterned, tentacles often strongly contrasting colour to disk

Zoanthus coppingeri

B Polyps immersed in thick coenenchyme. Intertidal on exposed reef crests. Colonies forming extensive rubbery mats. Colonies strikingly coloured, powder blue or green

Zoanthus vietnamensis 
thid populations on different individual reefs can be high (Burnett et al. 1994, 1995). Gene frequency differences should arise considerably faster than fixed differences, but larger sample sizes than ours would be required to reliably detect them.

\section{Identification of species in the field}

A dichotomous key to those members of the family Zoanthidae identified here is given in Table 5 , based on external characters which are easily interpreted in the field. The key includes Isaurus tuberculatus, not found during this study but previously reported from the GBR (Muirhead and Ryland 1985; Ryland and Muirhead 1993). Variation in features for visual identification of this species have not been evaluated by genetic means and diagnostic features are those of Muirhead (1989).

\section{Phylogeny and evolution of Zoanthidae}

Two main views of the phylogeny of the zoanthids have been proposed prior to the advent of genetic data. These views, in specific relation to the Zoanthidae and their relationship with Parazoanthus dichroicus, are illustrated in Fig. 4. The classical view of Haddon and Shackleton (1891) is based on mesenterial arrangement (Fig. 4a). The Zoanthidea are divided into suborders Macrocnemina (fifth dorsal mesentery perfect) and Brachycnemina (fifth dorsal mesentery imperfect). This view has been tacitly accepted by most workers including, most recently, Muirhead (1989). An alternative view by Schmidt (1974) is based on differences in the cnidom (Fig. 4b). Schmidt's "late Zoantharia" possess "special" microbasic b- mastigophores (nomenclature of England 1991) in the scapus. Genetic data provide a means of testing these two hypotheses. In genetic analyses, Parazoanthus dichroicus forms a natural outgroup (Fig. 2). Genetic distance measures show this species to be more distantly related to the Zoanthidae than members of the Zoanthidae are to one another. The use of this species as an outgroup in the maximum likelihood (CONTML) analysis therefore appears justified. The failure of $P$. dichroicus and Zoanthus spp. to form a monophyletic group refutes Schmidt's (1974) assertion that special microbasic b-mastigophores are a derived character of a (Parazoanthus, Zoanthus) clade. Instead, the genetically based phylogeny supports the classical systematic view of Haddon and Shackleton (1891).

The genetic analysis has shed light on two specific questions concerning the status of the genera Protopalythoa and Zoanthus. Firstly, from the data presented here the three Protopalythoa spp. cannot be treated as a monophyletic group and validity of the genus therefore appears doubtful. Genetic identities among all four Palythoa and Protopalythoa species are within the range suggested by Thorpe et al. (1978) for congeners. However, several physical features separate the two putative genera, particularly the number of tentacles (generally more in Protopalythoa spp. than in Palythoa spp., Ryland and

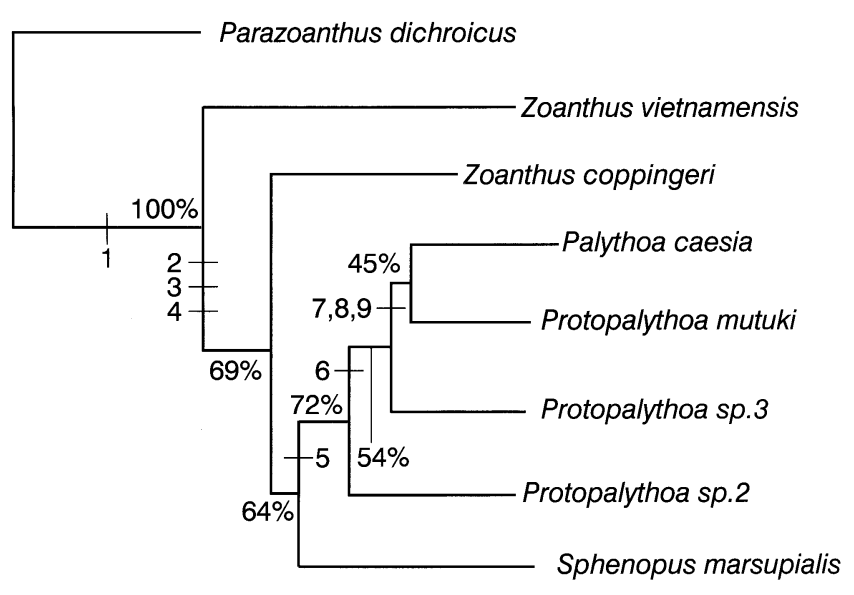

Key to markers:

1: $E S T{ }^{*} 100$; 2: LGG-2*100; 3: LT-*100; 4: LT-2*100; 5: IDH*100; 6: MDH-1*100; 7: GPI*115; 8: $G P I^{*} 100 ; 9:$ : $L G-1 * 112$

Fig. 3. Phylogeny of Zoanthidae using Parazoanthus dichroicus as a genetic outgroup. Estimated using a maximum likelihood method for gene grequencies which uses loci as characters with polymorphic states (CONTML, Felsenstein 1993). Percentiles indicate the number of times the group to the right occurred in the set of bootstrapped trees. Figures indicate alleles which are markers for the group to the right

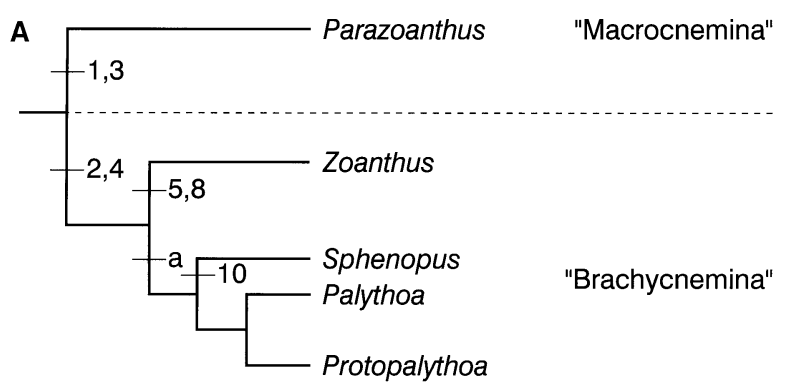

Primitive conditions: (1 or 2$),(3$ or 4$), 6,7,9, b$.

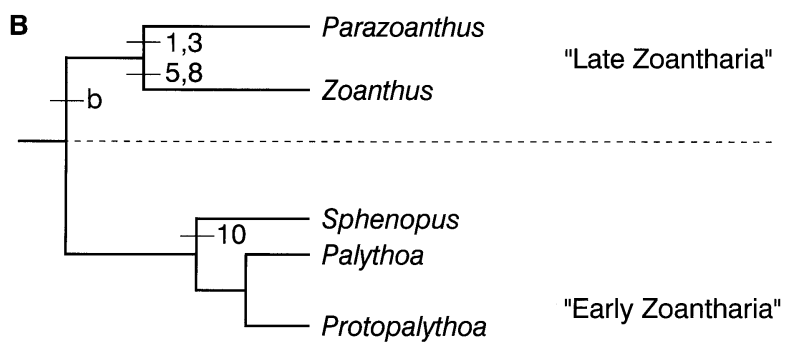

Primitive conditions: 2, 4, 6, 7, 9, a.

Key to characters:

1: Perfect 5th dorsal mesentery; 2: Imperfect 5th dorsal mesentery; 3 : Endodermal sphincter muscle; 4: Mesogloeal sphincter muscle; 5: Double sphincter muscle; 6 : Single sphincter muscle; 7: Sand encrusting; 8: Non-sand encrusting; 9: Colonial; 10: Solitary; a: No special microbasic b-mastigophores; b: Special microbasic b-mastigophores in scapus.

Fig. 4A, B. Zoanthidae. Views of the phylogeny of gtenera in the Zoanthidae and their relationship with Parazoanthus, based on morphological characters, by: A Haddon and Shackleton (1891); B Schmidt (1974). Figures denote morphological characters which fit the trees. The relative positions of the various genera are the same in the genetic based phylogeny (Fig. 3) as in $\mathbf{A}$ 
Muirhead 1993), and the extent to which polyps are embedded in the coenenchyme (completely in Palythoa spp., little if at all in Protopalythoa spp.). Furthermore, spawned ova from Pro. sp. 1 contain zooxanthellae (Ryland and Babcock 1991), while those from P. caesia do not (Burnett 1995b). Further work will be required to resolve the status of these two genera.

The two Zoanthus species do not appear as a monophyletic group in the phylogenetic analysis (Fig. 3). However, morphological criteria strongly suggest that Zoanthus species are closely related to one another relative to species in other genera. Hence there is a contradiction between genetic and morphological data with regards to the systematics of Zoanthus.

The genetic phylogeny is not well supported by bootstrap values, a consequence of a relatively low number of characters (14 loci) and large numbers of autapomorphies relative to synapomorphies. However, the tree is for the most part well supported by morphological characters, including mesenterial arrangement, sphincter muscle position, sphincter muscle structure (single or double) and sediment encrusting habit. Because of this, the genetic based phylogeny (Fig. 3) is likely to be an accurate, natural representation of systematics within the Zoanthidae. Less confidence is placed in the phylogeny in the two cases where genetic and morphological results are contradictory. A more powerful analysis would be helpful in resolving the questions concerning the two genera Zoanthus and Protopalythoa. Preliminary sequence data from 28S rDNA suggest that Protopalythoa is indeed monophyletic and should be considered a valid genus (Burnett 1995b).

In summary, allozyme electrophoresis has succeeded in delimiting seven groups of zoanthid morphs which are considered to be reproductively isolated species. The genetic data strongly suggest that a significant number of nominate zoanthid species represent morphotypes or ecotypes within a single species. Eight GBR species of Zoanthidae, including the most common and ecologically important, are now identifiable in the field. There is considerable morphological variation within species, the genetic basis of which remains to be determined. Genetic data suggest that the Zoanthidae as defined by Haddon and Shackleton (1891) are a valid natural group. Nematocyst data appear less useful in resolving systematic relationships within the order Zoanthidea than was suggested by Schmidt (1974). More genetic data are required to resolve problems with the systematics of the genera Protopalythoa and Zoanthus.

Acknowledgements. Thanks to C. Cartwright, A. Chen, M. Deslandis, T. Foyle, D. Garcia, M. Hall, J. Miller, R. Rowan, S. Sorokin, B. Stobart, S. Stobart, J. Walker, L. West, S. Williams and J. Yeatman for assistance in the field. D. Ayre, T. Hughes and two anonymous reviewers made many useful comments on the manuscript. Special thanks to the people of the Torres Strait for allowing collections from their traditional lands. Much of this work was heavily dependent on support from AIMS Marine Operations staff and the crews of the R. V.'s James Kirby, Harry Messell, Lady Basten and Sirius. This study was carried out during the tenure of NERC/CASE research studentship number GT4/91/ALS/47 to W.J.B. and is contribution number 804 from the Australian Institute of Marine Science.

\section{References}

Avise JC (1974) Systematic value of electrophoretic data. Syst Zool $23: 465-481$

Ayre DJ, Veron JEN, Duffy SL (1991) The corals Acropora palifera and Acropora cuneata are genetically and ecologically distinct. Coral Reefs 10:13-18

Babcock RC, Ryland JS (1990) Larval development of a tropical zoanthid (Protopalythoa sp.). Inv Repro Dev 17:229-236

Bucklin A, Hedgecock D (1982) Biochemical genetic evidence for a third species of Metridium (Coelenterata: Actiniaria). Mar Biol $66: 1-7$

Buth DG (1984) The application of electrophoretic data in systematic studies. Annu Rev Ecol Syst 15:501-522

Burnett WJ (1995a) Techniques for allozyme electrophoretic analysis of zoanthid samples. Aust Inst Mar Sci (AIMS) Rep Ser 20: $1-30$

Burnett WJ (1995b) Population structure and species delimitation of Zoanthidea. Ph D Thesis, University of Wales

Burnett WJ, Benzie JAH, Beardmore JA, Ryland JS (1994) High genetic variability and patchiness in a common Great Barrier Reef zoanthid (Palythoa caesia). Mar Biol 121:153-160

Burnett WJ, Benzie JAH, Beardmore JA, Ryland JS (1995) Patterns of genetic subdivision in populations of a clonal Cnidarian, Zoanthus coppingeri, from the Great Barrier Reef. Mar Biol $122: 665-673$

Carlgren O (1937) Ceriantharia and Zoantharia. Sci. Rep. Great Barrier Reef Expedition 1928-29 5:177-207

Carter MA, Thorpe JP (1981) Reproductive, genetic and ecological evidence that Actinia equina var. mesembryanthemum and var. fragacea are not conspecific. J Mar Biol Assoc UK $61: 79-93$

England KW (1991) Nematocysts of sea anemones (Actiniaria, Ceriantharia and Corallimorpharia: Cnidaria): nomenclature. Hydrobiologia 216/217: 691-697

Felsenstein J (1981) Evolutionary trees from gene frequencies and quantitative characters: finding maximum likelihood estimates. Evolution 35:1229-1242

Felsenstein J (1993) PHYLIP (Phylogeny Inference Package) version $3.5 \mathrm{c}$. Distributed by the author. Department of Genetics, University of Washington, Seattle

Garthwaite RL, Potts DC, Veron JEN, Done TJ (1994) Electrophoretic identification of poritid species (Anthozoa: Scleractinia). Coral Reefs 13:49-56

Goodall JA, Stoddart JA (1989) Techniques for the electrophoresis of mangrove tissue. Aquat Bot 35:197-207

Haddon AC, Shackleton AM (1891) Reports on the zoological collections made in Torres Straits by Professor A.C. Haddon, 1888-1889. Actiniae: I. Zoantheae. Sci Trans R. Soc Dublin 4: 673-701

Harris H, Hopkinson DA (1976) Handbook for enzyme electrophoresis in human genetics. North Holland, Oxford (loose-leaf)

Hertwig R (1882) Report on the Actiniaria dredged by HMS Challenger during the years 1873-1876. Report on the scientific results of the exploring voyage of HMS Challenger 1873-1876. Zoology $6: 1-122+$

Hertwig R (1888) Report on the Actiniaria dredged by HMS Challenger during the years 1873-1876. Supplement. Report on the scientific results of the exploring voyage of HMS Challenger 1873-1876. Zoology 26(78):4-56

Hillis DM (1987) Molecular versus morphological approaches to systematics. Annu Rev Ecol Syst 18:23-42

Karlson RH (1983) Disturbance and monopolisation of a spatial resource by Zoanthus sociatus (Coelenterata: Anthozoa). Bull Mar Sci 33:118-131

Lessios HA (1992) Testing electrophoretic data for agreement with Hardy-Weinberg expectations. Mar Biol 112:517-523

Levene H (1949) On a matching problem arising in genetics. Ann. Math Stat 20:91-94

Mayr E (1992) A local flora and the biological species concept. Am J Bot 79: 222-238 
Miller KJ, Benzie JAH (in press) No clear genetic distinction between morphological species within the coral genus Platygyra. Bull Mar Sci

Miller RG (1980) Simultaneous statistical inference. Springer-Verlag New York

Muirhead A (1989) Studies on Zoanthidea. Ph D Thesis, University of Wales

Muirhead A, Ryland JS (1985) A review of the genus Isaurus Gray, 1828 (Zoanthidea), including new records from Fiji. J Nat Hist $19: 323-335$

Nei M (1978) Estimation of average heterozygosity and genetic distance from a small number of individuals. Genetics $89: 583-590$

Poulik M (1957) Starch gel electrophoresis in a discontinuous system of buffers. Nature 180:1477-1479

Richardson BJ, Baverstock PR, Adams M (1986) Allozyme electrophoresis. Academic Press, Sydney

Ryland JS, Babcock RC (1991) Annual cycle of gametogenesis and spawning in a tropical zoanthid, Protopalythoa sp. Hydrobiologia 216/217: 117-123

Ryland JS, Muirhead A (1993) Order Zoanthidea. In: Mather P, Bennett I (eds) A coral reef handbook. Surrey Beatty and Sons, Chipping Norton, UK. pp 52-58

Saville-Kent W (1893) The Great Barrier Reef of Australia: its products and potentialities. WH Allen, London

Schmidt H (1974) On evolution in the Anthozoa. Proc 2nd Int Symp on Coral Reefs. 1:533-560

Shaw PW, Beardmore JA, Ryland JS (1987) Sagartia troglodytes (Anthozoa: Actiniaria) consists of two species. Mar Ecol Prog Ser $41: 21-28$

Smith BL, Potts DC (1987) Clonal and solitary anemones (Anthopleura) of western North America: population genetics and systematics. Mar Biol 94:537-546
Solé-Cava AM, Thorpe JP (1987) Further genetic evidence for the reproductive isolation of green sea anemone Actinia prasina Gosse from common intertidal beadlet anemone Actinia equina. Mar Ecol Prog Ser 38:225-229

Solé-Cava AM. Thorpe JP (1992) Genetic divergence between colour morphs in populations of the common intertidal sea anemones Actinia equina and A. prasina (Anthozoa; Actiniaria) in the Isle of Man. Mar Biol 112:243-252

Solé-Cava AM, Thorpe JP, Kaye JG (1985) Reproductive isolation with little genetic divergence between Urticina ( $=$ Tealia) felina and $U$. eques (Anthozoa: Actiniaria). Mar Biol 85:279-285

Stobart B, Benzie JAH (1994) Allozyme electrophoresis demonstrates that the scleractinian coral Montipora digitata is two species. Mar Biol 118: 183-190

Swofford DL, Selander RB (1981) BIOSYS-1: A FORTRAN program for the comprehensive analysis of electrophoretic data in population genetics and systematics. J Hered 72:281-283

Thorpe JP, Beardmore JA, Ryland JS (1978) Taxonomic intraspecific variation and genetic distance in the phylum Bryozoa. In: Battaglia B, Beardmore JA (eds.) Marine organisms: genetics, ecology and evolution. Plenum Press, New York, pp 425-445

Walsh GE, Bowers RL (1971) A review of Hawaiian zoanthids with descriptions of three new species. Zool J Linn Soc 50:161-180

Weil E (1992) Genetic and morphological variation in Caribbean and eastern Pacific Porites (Anthozoa, Scleractinia). Preliminary results. Proc 7th Int Coral Reef Symp Guam, 1992. 2:643-656

Wells JW, Hill D (1956) Zoanthinaria, Corallimorpharia, and Actiniaria. In: Moore RC (ed) Treatise on Invertebrate Paleontology. University of Kansas Press, Lawrence pp F232-F233

Wilsmore LJ (1909) On some Zoantheae from Queensland and the New Hebrides. J Linn Soc (Zool) 30:315-328

Wright S (1978) Evolution and the genetics of populations, vol 4. University of Chicago Press, Chicago USA 